\title{
A INFLUÊNCIA DAS IFRS NA ASSIMETRIA DOS CUSTOS DAS EMPRESAS BRASILEIRAS LISTADAS NA B3
}

\section{THE INFLUENCE OF IFRS ON THE ASYMMETRY OF COST OF BRAZILIAN COMPANIES LISTED IN B3}

Artigo apresentado no Congresso UFU de Contabilidade em 2019 e no Congresso AdCont 2019, no período de 17 e 18 de outubro de 2019 em Minas Gerais.

\section{RESUMO}

Este trabalho objetivou analisar o comportamento assimétrico dos custos e despesas em relação à receita, após adoção das International Financial Reporting Standards - IFRS, usando as informações de receita, custos e despesas de todas as empresas listadas na B3. O resultado apresenta similaridade com os já existentes na literatura, indicando assimetria no comportamento dos custos. A título de robustez, analisou-se a amostra com e sem as empresas financeiras e somente com as empresas financeiras. Considerando todas as empresas, após a adoção das IFRS, observou-se que o comportamento dos custos foi mais aderente. Ainda no período de adoção das IFRS, observou-se que o comportamento dos custos foi menos pegajoso, mesmo quando se separam as empresas em financeiras e não financeiras. Analisando somente as empresas financeiras com 376 observações, e comparando com as não financeiras, observou-se que os custos das empresas do setor financeiro não variam na mesma intensidade das receitas, apresentando, assim, um comportamento mais pegajoso, diferente dos apresentados nas literaturas já existentes.

Palavras-chave: Comportamento dos custos; IFRS; custo pegajoso.

\section{ABSTRACT}

This paper aims to analyze the asymmetric behavior of costs, and expenses in relation to revenue, after adoption of International Financial Reporting Standards (IFRS), using the revenue, cost and expense of all companies listed in B3. The result is similar to those found by prior literature, indicating asymmetry in cost behavior. For robustness, the sample was analyzed with and without financial companies and only with financial companies. Considering all companies, after the adoption of IFRS, it was observed that the behavior of costs was more sticky. Still in the IFRS adoption period, it was observed that the behavior of costs was less sticky, even when separating companies into financial and non-financial. Analyzing only the financial companies with 376 observations, and comparing with the non-financial ones, it was observed that the costs of the financial sector companies the is no variation in the same intensity of revenues, thus presenting a stickier behavior, different from those presented in prior literature.

Keywords: Cost behavior, IFRS; sticky cost.
Edelmira Rodrigues Costa

Mestre em Ciências Contábeis pela FUCAPE Business School. Contato: Av. Fernando Ferrari, 358 - Bairro Boa Vista CEP: 29.075-010- Vitória - ES - Telefone (27) 4009-4444

\section{Poliano Bastos da Cruz}

Doutor em Contabilidade e Administração FUCAPE Business School. Professor da FUCAPE. Contato: Av. Fernando Ferrari, 358 - Bairro Boa Vista - CEP: 29.075-010Vitória - ES- Telefone (27) 4009-4444

\section{Silvania Neris Nossa}

Doutora em Contabilidade e Administração FUCAPE Business School. Professora da FUCAPE e Presidente da Academia Capixaba de Contabilidade. Contato: Av. Fernando Ferrari, 358 - Bairro Boa Vista CEP: 29.075-010 - Vitória - ES -- Telefone (27) 4009-4444 ramal 4438 


\section{INTRODUÇÃO}

O presente estudo investiga a associação entre custo, despesas gerais e receita. Essa associação pode ser assimétrica, quando as variações dos custos e despesas gerais são diferentes em relação ao aumento e diminuição da receita, ou simétrica, quando as variações dos custos e despesas gerais têm a mesma proporção em relação ao aumento e diminuição da receita. Anderson, Banker e Janakiraman (2003) encontraram evidências de que os custos são assimétricos nas empresas Norte Americana, mostrando que os custos crescem, em média, 0,55\% para um aumento de 1\% na receita líquida de vendas, mas diminuem somente 0,35\% para uma redução de $1 \%$ na receita líquida de vendas.

Existe vasta literatura, no âmbito nacional e internacional, que evidencia o comportamento assimétrico dos custos nas empresas, ou seja, que aponta que os custos aumentam mais quando a receita aumenta do que diminuem quando a receita diminui (Medeiros, Costa, \& Silva, 2005; Richartz, Borgert, \& Lunkes, 2014; Grejo, Santos, \& Abbas, 2015; Banker \& Byzalov, 2014; Balakrishnan, Labro, \& Loderstrom, 2010; Banker, Byzalov, Ciftci, \& Mashruwala, 2014). Nesse sentido, Banker e Byzalov (2014) enfatizam que o comportamento assimétrico dos custos é um fenômeno global.

Em específico, Calleja, Steliaros e Thomas (2006) demonstraram que os custos nas empresas na França, na Alemanha, no Reino Unido e nos Estados Unidos (EUA) são assimétricos, pois aumentam, em média, de 0,97\% a 1\% na receita, mas diminuem apenas $0,91 \%$ para uma redução de $1 \%$ na receita. Os autores indentificaram ainda que os custos se mostram mais sticky (pegajoso) nas empresas francesas e alemãs, do que os custos das empresas do Reino Unido e dos EUA. Seguindo lógica similar, Richartz (2016) encontrou evidências de que as empresas brasileiras possuem comportamento assimétrico dos custos, similar aos encontrados na literatura internacional. Contudo, o autor excluiu da amostra as empresas do setor financeiro, fundos e outros no período investigado de 20 anos (1995 a 2014). Assim, o presente trabalho investigou a mesma questão, porém ora mantendo as empresas do setor financeiro, ora excluindo-as, pesquisou também somente as empresas do setor financeiro e ainda a adoção completa das IFRS, com vistas a gerar maior robustez aos resultados.

Com base na revisão de literatura, identificou-se que a adoção do International Financial Reporting Standards (IFRS) pode ter influenciado no comportamento assimétrico dos custos (Bugeja, Lu, \& Shan, 2015), visto que algumas despesas como a depreciação sofreram mudanças no seu reconhecimento. No Brasil, a adoção completa das IFRS aconteceu em 2010, por meio da emissão dos Comitês de Pronunciamentos Contábeis (CPC). Não foram identificados, na literatura nacional, estudos que investiguem o efeito da adoção das IFRS no comportamento assimétrico dos custos, tendo sido identificado apenas o estudo de Bugeja, Lu e Shan (2015), na literatura internacional, que investigou a questão na Austrália.

O presente trabalho defende que é possível que essas características somadas à adoção das IFRS tenham impactado o comportamento dos custos, o que torna o Brasil um caso interessante para estudo da assimetria dos custos.

Diante do exposto, o presente estudo tem como problema de pesquisa a seguinte pergunta: a adoção das IFRS impactou no comportamento assimétrico dos custos?

Com vistas a responder o problema de pesquisa, seguiu-se o modelo empírico proposto por Anderson et al. (2003), que possibilita a medição dos custos e despesas em relação às mudanças na receita de vendas e discrimina, entre períodos, quando a receita diminui e quando ela aumenta. Para isso, utilizou-se de informações das demonstrações contábeis, receitas, custos, despesas de venda e administrativas de todas as empresas listadas na B3. Foram excluídas apenas observações referentes às empresas que não apresentaram alguma das informações necessárias para operacionalização do estudo. O período investigado foi de 16 anos, entre 2000 a 2016, antes da adoção completa das IFRS (2000 a 2009 ) e pós-adoção completa das IFRS (2010 a 2016).

Espera-se que este estudo traga contribuições teóricas para a literatura, visto que não se identificou uma quantidade significativa de trabalhos que investiguem o efeito das IFRS na assimetria dos custos. Já a contribuição prática de se conhecer o efeito da adoção das IFRS no comportamento dos custos acaba sendo crucial para o processo de tomada de decisão dos investimentos, tendo em vista que, nos dias atuais, a gestão estratégica de custo tem auxiliado as organizações na tomada de decisões.

\section{REFERENCIAL TEÓRICO}

\subsection{Comportamento dos Custos}

Segundo Anderson (2006), a gestão estratégica dos custos assume duas formas: a primeira trata-se da gestão estrutural dos custos que emprega ferramentas de organização, de produto e de processos para construir uma estrutura de custos coerente com a estratégia. Já a segunda, refere-se ao gerenciamento de custos executivos que emprega várias ferramentas de medição e análise (e.g., análise de custo e de variância) para avaliar o desempenho dos custos, permitindo a estruturação de um modelo que relaciona gerenciamento estratégico de custos com desenvolvimento de estratégia e avaliação de desempenho. Ambas as formas citadas constituem o gerenciamento estratégico de custos, atraindo interesse de gestores e acadêmicos na aquisição de habilidades de gerenciamento de custos.

Corroborando esta ideia, Medeiros et al. (2005) dizem que o conhecimento do comportamento dos custos é de extrema importância, tanto para acadêmicos quanto para profissionais que atuam diretamente na gestão empresarial. O conhecimento dos custos é um dos pilares para tomada de decisões gerenciais e, consequentemente, entender o seu comportamento diante de variações nas receitas pode ser uma fonte de competitividade para as empresas. 
Sendo assim, para compreender o comportamento assimétrico dos custos, é importante identificar a sua fonte. Nesse sentido, Anderson et al. (2003) identificaram que alguns custos acabam tendo seu comportamento impactado pela decisão dos gestores. Isso decorre do fato de que o comportamento dos custos não depende apenas da variação do volume de atividades (e.g., dispensar ou não funcionários quando as atividades diminuem) evidenciando que as variações nos custos não se resumem em variações nos custos fixos e variáveis, como apregoado nos modelos tradicionais. Concordando com Anderson et al. (2003), Medeiros et al. (2005) constataram que a gestão pode verificar e controlar assimetria do custo, podendo observar a sensibilidade dos custos em relação ao volume e tomar decisões contratuais. Esses custos são ajustados aos níveis de recursos contratados e de acordo com as decisões dos gerentes que tenham interesse de gerenciar os resultados da empresa.

Tratando ainda de custos de agência, a assimetria de custo está positivamente correlacionada aos incentivos de construção de impérios gerenciais. Além disso, foi verificado que a correlação positiva entre custo de agência e a assimetria de custos é mais acentuada sob a governança corporativa, que desempenha um papel importante nos efeitos dos problemas de agência e nas decisões de ajuste dos custos (Chen, Lu \& Sougiannis, 2008).

Kama e Weiss (2013) também constataram que problemas de agência podem ser os possíveis ocasionadores da assimetria. $\mathrm{O}$ argumento desses autores é de que as motivações para ajustes de recursos por parte dos gestores, com vistas a cumprir metas de lucros, bem como os incentivos dos gerentes em evitar perdas ou atender previsões de ganhos, impactam no comportamento dos custos. Neste sentido Silva, Zonatto e Klan (2019) concluíram que os accruals totais e parte do lucro são relacionados aos custos que se comportam de forma assimétrica. Ainda, Kama e Weiss (2013) trazem outros elementos que influenciam no comportamento assimétrico dos custos, como os gastos com a mão de obra que sofrem resistências dos gestores em extinguir esse recurso em momento de declínio de vendas, pois creem que a queda pode ser temporária e logo o volume retornará ao patamar inicial, evitando, assim, a necessidade de contratação e treinamento. Além disso, outro elemento seria o grau de participação dos custos fixos na composição do custo total, pois os custos fixos demoram mais para serem ajustados.

Já para Richartz, Borgert e Lunkes (2014) e Elias, Borgert e Richartz (2013), os gastos com mão de obra não influenciam no comportamento assimétrico dos custos totais das empresas brasileiras. Além disso, percebe-se ainda que a assimetria é um pouco maior nas empresas com maior participação de custo fixo. Neste sentido, Dierynck, Landsman e Renders (2012) demonstram os resultados obtidos nas empresas privadas da Bélgica, com incentivos para atingir ou superar a meta de lucro igual a zero, limitando o aumento dos custos de mão de obra, mesmo após um aumento nas atividades. Todavia elas estão dispostas a cortar os custos de mão de obra após uma diminuição da atividade. As empresas que não enfrentam uma pressão significativa do benchmark de ganhos demonstram um comportamento assimétrico dos custos de mão de obra.

Banker, Byzalov e Chen (2013), ao realizarem a pesquisa em dezenove países, perceberam que as empresas, ao forneceram proteção mais rigorosa ao emprego, apresentaram maior grau de aderência ao custo. Observa-se também, nesse estudo, que o comportamento de custo observado reflete em decisões deliberadas de comprometimento de recursos por gestores que enfrentam custo de ajuste.

Partindo de lógica similar, Balakrishnan, Labro e Loderstrom (2010) evidenciaram que a ação dos gerentes, tanto em curto prazo quanto em longo prazo, interfere no comportamento dos custos. Em longo prazo, os gerentes possuem controle considerável sobre a estrutura de custos, mesmo com algumas restrições impostas, como o crescimento do PIB e os custos fixos. Contudo Banker et al. (2014) enfatizam que os custos são componentes importantes dos ganhos e reconhecem explicitamente o impacto das expectativas dos gestores sobre o comportamento dos custos.

Por sua vez, Weiss (2010) enfatiza que a rigidez dos custos influencia as prioridades de cobertura dos analistas e dos investidores, que consideram o comportamento de custo sticky (pegajosos) na formação de suas crenças sobre o valor das empresas. Nesse mesmo sentido, Porporato e Werbin (2010) aplicaram o conceito dos Sticky Costs (custo pegajoso) em bancos localizados na Argentina, Canadá e Brasil, entre os anos de 2004 a 2009, cujos resultados sugeriram que os bancos com maiores proporções de custos fixos, como os do Brasil, apresentam menor redução de custos quando a receita diminui. Enquanto isso, os bancos com os níveis mais elevados de custos variáveis (Canadá) tiveram maior redução diante das diminuições da demanda.

Calleja et al. (2006) visualizaram que os custos nas empresas da França, Alemanha, Reino Unido e dos EUA são assimetricos, pois aumentam, em média, de 0,97\% para 1\% na receita, mas diminuem apenas 0,91\% para uma redução de $1 \%$ na receita. Os autores indentificaram ainda que os custos que apresentam o seguinte comportamento são mais sticky (pegajoso) nas empresas francesas e alemãs do que os custos das empresas inglesas e estadounidenses.

Nesse sentido, alguns trabalhos investigaram empresas de um setor específico, como Grejo, Santos e Abbas (2015), que salientam as empresas de Tecnologia da Informação, cujo Custo do Produto Vendido (CPV) absorve, em média, 0,63\% para $1 \%$ da Receita Líquida de Venda (RLV), apresentando existência de um comportamento assimétrico dos custos.

Sob a mesma ótica, Richartz (2016) apresenta resultados encontrados de alguns estudos, como hipóteses que foram testadas em sua tese de doutorado, sendo esses resultados defendidos pelo autor como os mais relevantes na literatura: a) estrutura de custos das empresas; b) fluxo de caixa disponível; c) tamanho da empresa; d) intensidade de ativos e passivos; e) decisões deliberadas dos gestores; f) ambiente macroeconômico; g) magnitude das variações da receita; h) legislação de proteção ao emprego; i) regulamentação do mercado; j) atraso nos ajustes de custos; l) problemas de agência. A pesquisa conclui ainda que os resultados do Brasil são similares aos encontrados na literatura internacional. 
Com base na discussão acima, o presente estudo tenta verificar as seguintes hipóteses:

$\mathbf{H}_{\mathbf{1}}$ : Aumento nos custos e despesas de vendas e administrativas para um aumento na receita é superior à diminuição nos custos e despesas de vendas e administrativas para uma redução na receita.

$\mathbf{H}_{1 \mathbf{a}}$ : Para empresas financeiras, o aumento nos custos e despesas de vendas e administrativas para um aumento na receita é superior à diminuição nos custos e despesas de vendas e administrativas para uma redução na receita.

\subsection{Adoção das IFRS}

Telles e Salotti (2015) levantam uma questão que pode ser relevante para o comportamento assimétrico dos custos. Os autores argumentam que a falta do entendimento das normas contábeis faz com que as empresas reconheçam erroneamente a despesa com depreciação. Isso pode impactar de várias formas no resultado das firmas. Por exemplo, podem-se ter dois carros: um para fins particulares e outro para aluguel (renda). Antes, a depreciação era feita de forma igual, agora irá variar, já que um sofre mais desvalorização econômica do que o outro. Dados esses elementos, argumenta-se que a adoção das IFRS pode afetar no comportamento assimétrico dos custos (Bugeja et al., 2015).

Sobre a CPC 27, Domingos, Lima e Pontes (2013) pesquisaram as 100 maiores companhias listadas na edição de 2012 da Revista Exame e na B3, no período de 2008 a 2011. Por meio dessa pesquisa, os autores observaram que, após adoção das IFRS, as empresas passaram a utilizar as contas de despesa com depreciação, outras receitas operacionais e provisões de curto prazo para gerenciar os resultados contábeis, visando reduzir a variabilidade do lucro.

Por outro lado, o CPC 16 estabelece que os estoques devam ser mensurados pelo valor de custo ou pelo valor realizável, considerando-se, entre os dois, o menor. Esse custo deve incluir os custos de compra como o preço de compra, os impostos não recuperáveis, transporte, seguro, manuseio e outros custos atribuíveis às aquisições de produtos, mercadorias, materiais e serviços. Além disso, o CPC 16 traz que os custos de transformação incluem todos os gastos que estejam relacionados às unidades produzidas ou à linha de produção, como os gastos diretos e indiretos, fixos e variáveis que sejam incorridos para transformar os materiais em produtos acabados.

Lourenço e Branco (2015) observaram que, em regra geral, a adoção das IFRS apresenta um efeito positivo na qualidade da informação, no mercado de capitais, na capacidade de previsão dos analistas, na comparabilidade e no uso da informação. Contudo, esse efeito depende de alguns fatores, como as características dos países e das empresas.

Enquanto Santos e Cavalcante (2014) concluíram que, com a adoção das IFRS no Brasil, observou-se um aumento na capacidade associativa do lucro contábil, não foi significativa a tempestividade informacional e também não surtiu efeito sobre o conservadorismo condicional, quando analisado em conjunto. Conclui-se que não é possível afirmar que a adoção das IFRS no Brasil contribuiu com o aumento da relevância informacional do lucro contábil das firmas de capital aberto.

Por sua vez, Gatsios, Silva, Ambrozini, Assaf e Lima (2016) indicam que a adoção das IFRS não contribui para a redução dos custos de capital das empresas pesquisadas. Isso sugere que o processo de adoção pode levar mais tempo para impactar no custo de capital das empresas brasileiras de capital aberto, pois o impacto da adoção das IFRS não está somente com as normas, mas com o uso pelas empresas e demais usuários.

Autores como Silva, Bonfim, Niyama e Silva (2017) abordam uma relação significante e positiva entre a adoção das IFRS e a qualidade do lucro das empresas listadas na B3. Já Silva e Miranda (2016) pesquisaram em 35 setores da economia, no período de quatro anos antes e quatro anos após a adoção das IFRS, sendo observado que o capital de giro em elementos operacionais e financeiros foi mais impactado pela a adoção das IFRS. Partindo de lógicas similares, Sousa, Sousa e Demonier (2016) pesquisaram os demonstrativos financeiros de 320 empresas listadas na B3, no período de 2000 a 2012, sendo observado que a adoção completa das IFRS não impactou no grau de conservadorismo nos demonstrativos financeiros analisados.

Nesse sentido, é observado que a maior parte dos estudos que investigaram os efeitos da adoção das IFRS tem tratado dos efeitos dessa adoção na qualidade da informação contábil. Uma exceção é o estudo realizado por Bugeja et al. (2015). Bugeja et al. (2015) pesquisaram as empresas australianas que apresentaram um comportamento em que os custos aumentaram $0,885 \%$ com um aumento de $1 \%$ em receitas de vendas, mas diminuíram apenas 0,797\% para uma queda de $1 \%$ nas receitas de vendas. Ainda observaram que aderência dos custos aumentou após a adoção das IFRS. Contudo, o comportamento dos custos é mais pegajoso para serviços e indústrias e não é significativo para a construção e varejo. Como pode ser visto nesta revisão da literatura, não se identificou quantidade significativa de estudos que investigam o efeito das IFRS na assimetria dos custos. Em específico, no Brasil, não se identificou nenhum estudo que investigue o efeito das IFRS na assimetria dos custos. Após serem analisados e discutidos os fatores, o presente estudo tenta verificar a seguinte hipótese:

$\mathbf{H}_{2}$ : A adoção completa das IFRS aumentou o comportamento assimétrico dos custos.

\section{METODOLOGIA}

\subsection{Natureza da pesquisa}

A presente pesquisa utilizou o modelo empírico proposto por Anderson et al. (2003) para estimar a assimetria dos custos em empresas brasileiras. Esse método mostrou-se eficaz na mensuração da assimetria dos custos em relação à re- 
ceita para empresas norte-americanas e já foi utilizado em vários estudos, tanto nacionais como internacionais (Medeiros et al., 2005; Richartz et al., 2014; Grejo et al., 2015; Banker \& Byzalov, 2014; Balakrishnan et al., 2014; Banker et al., 2014).

Foram estabelecidos dois modelos econométricos para investigação das hipóteses. O Modelo 1 fornece base para o teste das variações dos custos, despesas de vendas e administrativas em relação à receita, sendo utilizado para testar as $\mathbf{H}_{1 \mathrm{e}} \mathbf{H}_{1 \mathrm{a}}$.

A equação (1) mostra o efeito da variação na Receita nas variações no CDVA.

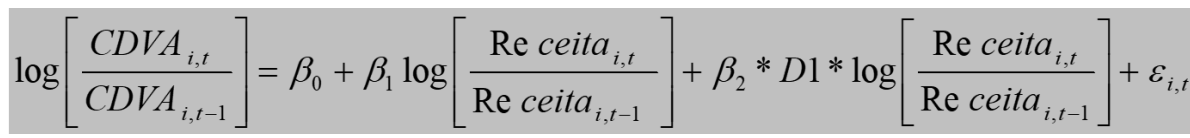

O i significa a empresa; t indica o tempo; Ei,t é o termo de erro; log o logaritmo natural. As variáveis estão listadas no Quadro 1.

Em específico, o coeficiente $\beta 1$ mede o percentual de aumento no CDVA relativo a um aumento de $1 \%$ na Receita das empresas. Já o coeficiente $\beta 2$ mede o percentual de redução no CDVA relativo a uma redução de $1 \%$ na Receita. Se o CDVA possuir variação assimétrica, sua variação relativa ao aumento da Receita deve ser maior que a variação relativa à redução da Receita.

Quadro 1 - Composição das variáveis da pesquisa para modelo I e II

\begin{tabular}{|c|c|c|}
\hline Sigla & Variável dependente & \\
\hline$\left[\frac{C D V A_{i, t}}{C D V A_{i, t-1}}\right]$ & \multicolumn{2}{|c|}{$\begin{array}{l}\text { CDVA (custo despesas de venda e administrativa) do período dividido pelo CDVA (custo despesas de } \\
\text { venda e administrativa) do período anterior custo, despesas de venda e administrativa. }\end{array}$} \\
\hline Siglas & Variáveis independentes & Sinal esperado \\
\hline$\frac{\operatorname{Re} c e i t a_{i, t}}{\operatorname{Re} \text { ceita }_{i, t-1}}$ & Variações da receita do período dividido pela receita do período anterior. & + \\
\hline D1 & $\begin{array}{l}\text { Dummy de diminuição da receita tem valor de }(1) \text { quando a receita da empresa } \\
\text { (i) no tempo }(\mathrm{t}) \text { diminui relativo ao período }(\mathrm{t}-1) \text { e assume o valor de }(0) \text { quando } \\
\text { a receita aumenta. }\end{array}$ & - \\
\hline IFRS & $\begin{array}{c}\text { (após a adoção das IFRS), tendo valor de (0) para os anos de } 2000 \text { a } 2009 \text { e } \\
\text { valor de (1) para os anos de } 2010 \text { a } 2016 .\end{array}$ & - \\
\hline
\end{tabular}

Fonte: Elaboração própria.

O Modelo 2 também fornece base para teste das variações dos CDVA relativos a variações na Receita, só que controlando a adoção das IFRS. Com esse modelo, testou-se a $\mathbf{H}_{2}$.

$$
\begin{aligned}
& \log \left[\frac{C D V A_{i, t}}{C D V A_{i, t-1}}\right]=\beta_{0}+\beta_{1} \log \left[\frac{\operatorname{Re} \text { ceita }_{i, t}}{\operatorname{Re} \text { ceita }_{i, t-1}}\right]+\beta_{2} * D 1 * \log \left[\frac{\operatorname{Re} \text { ceita }_{i, t}}{\operatorname{Re} \text { ceita }_{i, t-1}}\right] \\
& +\beta_{3} * D 1 * \log \left[\frac{\operatorname{Re} \text { ceita }_{i, t}}{\operatorname{Re} \text { ceita }_{i, t-1}}\right] * \operatorname{IFRS}+\varepsilon_{i, t}
\end{aligned}
$$

O i significa a empresa; t indica o tempo; Ei,t é o termo de erro; log o logaritmo natural. As variáveis estão listadas no Quadro 1.

A Equação 2 mostra o efeito da variação na Receita nas variações no CDVA. Em específico, os coeficientes $\beta 1$ e $\beta 2$ possuem interpretação análoga ao Modelo 1. Por outro lado, o coeficiente $\beta 3$ mede o percentual de redução do CDVA relativo a uma redução de $1 \%$ na receita após a adoção das IFRS. Se o CDVA possuir variação assimétrica mais acentuada após a adoção das IFRS, a variação relativa ao aumento da Receita $(\beta 1)$ deve ser maior que a variação relativa à redução da Receita ( $\beta 2)$, somada ao coeficiente $\beta 3$, que se espera ter coeficiente negativo. 


\subsection{Amostra}

Para atender o objetivo da pesquisa e responder as hipóteses, foram coletados os dados dos anos de 2000 a 2016 , analisados em painel. A amostra foi composta por 6.598 observações para período definido, no qual foram consideradas todas as empresas que apresentaram as variáveis necessárias para a realização da pesquisa, sendo estas: receita, custo, despesas de venda e administrativa. As variáveis foram winsorizadas para mitigar o efeito de outliers. Os valores considerados foram de 0,025\%, no limite inferior, e 0,025\%, no limite superior. Os desvios-padrão da amostra winsorizada se mostraram menores.

Na Tabela 1, é apresenta a estatística descritiva, a qual permite que seja verificado como os dados CDVA e as receitas se comportam.

Tabela 1 - Estatística descritiva

\begin{tabular}{c|c|c|c|c|c}
\hline Variáveis & Observações & Média & Desvio Padrão & Mínimo & Máximo \\
\hline CDVA & 6598 & $2,5 \mathrm{e}+06$ & $1,0 \mathrm{e}+07$ & 4,00 & $2,8 \mathrm{e}+08$ \\
Receita & 6598 & $3,0 \mathrm{e}+06$ & $1,3 \mathrm{e}+07$ & 1,00 & $3,4 \mathrm{e}+08$ \\
$\Delta$ CDVA & 6598 & 0,10 & 0,49 & $-7,47$ & 7,25 \\
$\Delta$ CDVAw & 6598 & 0,10 & 0,30 & $-0,74$ & 0,98 \\
$\Delta$ Receita & 6598 & 0,09 & 0,62 & $-8,76$ & 7,29 \\
$\Delta$ Receitaw & 6598 & 0,10 & 0,33 & $-0,88$ & 1,08 \\
\hline
\end{tabular}

Esta tabela contém as estatísticas descritivas da amostra, a variável CDVA são os custos e despesas de venda e administrativa, e a variável receita são todas as receitas, $\triangle \mathrm{CDVA}$ é taxa da variação, e $\Delta$ Receita é a taxa da variação.

Fonte: Elaboração própria.

Observou-se que a média apresenta um valor nominal para CDVA de 2,5e+06, e para receita de 3,0e+06. Portanto, custo, despesas de venda e administrativa foram levemente inferiores à receita no período de análise. Porém, observa-se que a variação da taxa para o CDVA foi de 10\%, enquanto a média na variação da taxa para receita foi de $9 \%$. Assim, custo, despesas de venda e administrativa foram levemente superiores à receita no período de análise. Quando as variáveis são winsorizadas, apresentam um resultado na taxa média igual para ambos de $10 \%$. Por outro lado, o desvio padrão apresenta um valor nominal para CDVA de 1,0e+07, e para receita de 1,3e+07, também assumindo valores de variação elevados para ambas as variáveis em estudo. Porém observa-se que a variação da taxa para CDVA foi de 4,9\% e para receita foi de 6,2\%. No entanto, após winsorizar, as variáveis apresentam desvios-padrão menores para CDVA de 3,0\% e para receita de 3,3\%.

$\mathrm{Na}$ Tabela 2, é apresentada a correlação entre as variáveis, considerando a amostra com todas as empresas, totalizando 6.598 observações.

O resultado da correlação que consta na Tabela 2 abaixo entre as variáveis, receita e CDVA apresentam correlação positiva forte de 0,9883. Ou seja, havendo aumento da receita, aumenta o CDVA. Quando são analisados o CDVA e a receita, observa-se que a taxa de variação da receita exibe correlação positiva forte de 0,7657 com a variável CDVA, sendo contínua no presente e no futuro e diretamente proporcional à sua relação. No entanto, após winsorizar, as variáveis apresentam menor correlação positiva, porém ainda forte: 0,7518. O grau positivo e forte da correlação aponta que à medida que a receita aumenta os CDVA também aumentam e, quando a receita diminui, os CDVA também diminuem a tendência de elevação a qual encontra respaldo nas premissas previstas do escopo deste trabalho.

Tabela 2 - Correlação

\begin{tabular}{c|c|c|c|c|c|c}
\hline & CDVA & Receita & CDVA $_{\mathrm{i}, \mathrm{t}-1}$ & Receita $_{\mathrm{i}, \mathrm{t}-1}$ & CDVA $_{\mathrm{i}, \mathrm{t}-\mathbf{1} \mathbf{w}}$ & Receita $_{\mathrm{i}, \mathrm{t}-1 \mathbf{w}}$ \\
\hline CDVA & 1,0000 & & & & \\
Receita & 0,9883 & 1,0000 & & & \\
CDVA $_{\mathrm{i} t \mathrm{t}-1}$ & 0,0173 & 0,0160 & 1,0000 & & \\
Receita $_{\mathrm{i}, \mathrm{t}-1}$ & 0,0123 & 0,0135 & 0,7657 & 1,0000 & & \\
CDVA $_{\mathrm{i}, \mathrm{t}-\mathrm{w} w}$ & 0,0250 & 0,0234 & 0,8383 & 0,6107 & 1,0000 & \\
Receita $_{\mathrm{i}, \mathrm{t}-\mathrm{w}}$ & 0,0178 & 0,0200 & 0,6531 & 0,8052 & 0,7518 & 1,0000 \\
\hline
\end{tabular}

Esta tabela contém as correlações entre as variáveis, sendo que CDVA são os custos e despesas de venda e administrativa, receita são todas as receitas. CDVA $_{\mathrm{i}^{\prime} \mathrm{t}-1}$ é o custo despesas de venda e administrativa do tempo atual dividida pelo tempo anterior e Receita $\mathrm{i}_{\mathrm{i},-1}$ é receita do tempo atual dividida pelo tempo anterior. CDVA $_{\text {it-1w }}$ é o custo e despesas de venda e administrativa do tempo atual dividida pelo tempo anterior winsorizados e receitai ${ }_{t-1 \mathrm{w}}$ é receita do tempo atual dividida pelo tempo anterior winsorizada.

Fonte: Elaboração própria. 


\section{RESULTADOS}

Os resultados das regressões são apresentados e discutidos a seguir. As estimações foram realizadas pelo estimador de Efeitos Fixos (EF) e Efeitos Aleatórios (EA), conforme o teste de Hausman. Para computar o efeito líquido da redução na receita, ao contrário da literatura que apenas soma os coeficientes $\beta 1$ e $\beta 2$, conduziu-se um teste de hipótese de combinação linear equivalente a um teste $\mathrm{F}$.

Tabela 3 - Teste de Hausman para modelo I com todas as empresas

\begin{tabular}{c|c}
\hline & Coef. \\
\hline Chi-square test value & 6.2 \\
P-value & $.045^{\star *}$ \\
\hline
\end{tabular}

Esta tabela contém as regressões para todas as amostras.

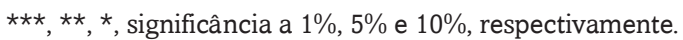

Fonte: Elaboração própria.

Na Tabela 3 é apresentada a estatística qui-quadrado do Teste de Hausman para o modelo I com todas as empresas. Nota-se pelo p-valor que é rejeitada a hipótese nula de que a diferença de coeficientes estimados por meio de EF e EA é não sistemática, indicando que o estimador adequado é o de EF. Na Tabela 3.1, apresentam-se os resultados para o Modelo 1, considerando a amostra com todas as empresas, totalizando 6.598 observações. Observou-se que o aumento dos custos e despesas de vendas e administrativas para um aumento da receita é superior à diminuição dos custos e despesas de vendas e administrativas para uma redução da receita. Portanto, não se rejeita a $\mathrm{H}_{1}$. Desta forma, os resultados encontrados apontam que, quando a receita aumenta $1 \%$, os custos e despesas aumentam $0,73 \%$. Porém, quando a receita diminui $1 \%$, esses mesmos custos e despesas reduzem $0,61 \%(0,7304-0,1221)$. Ou seja, os custos e despesas das empresas brasileiras possuem comportamento assimétrico, similar aos encontrados na literatura sobre o assunto.

Tabela 3.1 - Análises do comportamento dos custos para modelo I com todas as empresas

\begin{tabular}{|c|c|c|c|c|}
\hline Variáveis & Coeficiente & Desvio Padrão & Estatística T & P-Valor \\
\hline Receita & 0,7304 & 0,0110 & 65,86 & $0,000 * \star \star *$ \\
\hline D1 & $-0,1221$ & 0,0199 & $-6,12$ & $0,000 * * *$ \\
\hline Constante & 0,0191 & 0,0032 & 5,82 & 0,000 \\
\hline \multicolumn{5}{|c|}{$\begin{array}{c}\text { Observances }=6.598 \\
\text { R-sq.: Within }=0,5579 \\
\text { Between }=0,9412 \\
\text { Overall }=0,5677\end{array}$} \\
\hline
\end{tabular}

Esta tabela contém as regressões para todas as amostras. CDVA, variável dependente, são os custos e despesas de venda e administrativa, receita são todas as receitas, D1 quando a receita diminui, variação do indicador tem valor de (1) quando a receita da empresa (i) no tempo $(t)$ diminui para período $(t-1)$ e valor de $(0)$ quando a receita aumenta. ${ }^{* \star *}$, **, , , significância a $1 \%, 5 \%$ e $10 \%$, respectivamente.

Fonte: Elaboração própria.

O trabalho de Anderson et al. (2003), por exemplo, mostra que os custos aumentam em média 0,55\% para um aumento de $1 \%$ da receita líquida de vendas, mas diminuem somente $0,35 \%$ para uma redução de $1 \%$ da receita líquida de vendas. O resultado da pesquisa de Calleja et al. (2006) sugere um aumento de, em média, 0,97\% dos custos por aumento de $1 \%$ da receita, mas diminui apenas $0,91 \%$ por diminuição de $1 \%$ da receita.

Tabela 3.2 - Efeito líquido da assimetria dos custos

\begin{tabular}{c|c|c|c|c}
\hline Variáveis & Coeficiente & Desvio Padrão & Estatística T & P-Valor \\
\hline Efeito do aumento receita & 0,7304 & 0,0110 & 65,86 & $0,000^{\star \star \star}$ \\
Efeito da diminuição receita & 0,6083 & 0,0139 & 43,59 & $0,000^{\star \star \star}$ \\
\hline
\end{tabular}

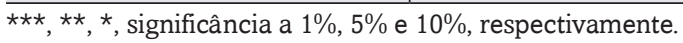

Fonte: Elaboração própria.

Na Tabela 4 é apresentada a estatística qui-quadrado do Teste de Hausman para o modelo II com todas as empresas. Nota-se pelo p-valor que é rejeitada a hipótese nula de que a diferença de coeficientes estimados por meio de EF e EA é não sistemática, indicando que o estimador adequado é o de EF. Na Tabela 4.1 apresentam-se os resultados para 
o Modelo 2 e também para a amostra total de empresas. Desta forma, os resultados da presente pesquisa apontam que, quando a receita aumenta $1 \%$, os custos e despesas aumentam $0,73 \%$, mas, quando a receita diminui $1 \%$, os custos e despesas reduzem $0,59 \%$ (0,7299-0,0965 - 0,0443). Tal valor é menor do que foi observado na Tabela 3, indicando que os custos são mais pegajosos após a adoção das IFRS.

Tabela 4 - Teste de Hausman para modelo II adoção comp. das IFRS

\begin{tabular}{c|c}
\hline & Coef. \\
\hline Chi-square test value & 6.598 \\
P-value & $.086^{*}$ \\
\hline
\end{tabular}

Esta tabela contém as regressões para todas as amostras.

$\star \star \star$, **, *, significância a $1 \%, 5 \%$ e $10 \%$, respectivamente.

Fonte: Elaboração própria.

Esse resultado corrobora a pesquisa de Bugeja et al. (2015), que analisaram as empresas australianas que apresentaram um comportamento em que os custos aumentaram 0,88\% com um aumento de $1 \%$ em receitas de vendas, mas diminuíram apenas $0,80 \%$ para uma queda de $1 \%$ nas receitas de vendas, e, ainda, observaram que os custos são mais pegajosos após a adoção das IFRS.

Tabela 4.1 - Análises do comportamento dos custos para modelo II adoção comp. das IFRS

\begin{tabular}{|c|c|c|c|c|}
\hline Variáveis & Coeficiente & Desvio Padrão & Estatística T & P-Valor \\
\hline Receita & 0,7299 & 0,0110 & 65,80 & $0,000 * \star \star$ \\
\hline D1 & $-0,0965$ & 0,0253 & $-3,80$ & 0,000 *** \\
\hline D1*IFRS & $-0,0443$ & 0,0271 & $-1,63$ & 0,103 \\
\hline Constante & 0,0192 & 0,0032 & 5,85 & 0,000 \\
\hline \multicolumn{5}{|c|}{$\begin{array}{c}\text { Observances }=6.598 \\
\text { R-sq: Within }=0,5581 \\
\text { Between }=0,9416 \\
\text { Overall }=0,5678\end{array}$} \\
\hline
\end{tabular}

Esta tabela contém as regressões para todas as empresas: CDVA, a variável dependente, são os custos e despesas de venda e administrativa, receita são todas as receitas, D1 quando a receita diminui, variação do indicador tem valor de 1 quando a receita da empresa i no tempo t diminui para período $\mathrm{t}-1$ e valor de 0 quando a receita aumenta, D1*IFRS após adoção das IFRS variação do indicador tem valor de 0 nos anos 2000 a 2009 e valor de 1 para os anos 2010 a 2016.

***, **, *, significância a 1\%,5\% e 10\%, respectivamente.

Fonte: Elaboração própria.

Observou-se que o aumento dos custos e despesas de vendas e administrativas para um aumento da receita é superior à diminuição dos custos e despesas de vendas e administrativas para uma redução da receita. Com isso, não se rejeita a $\mathrm{H}_{2}$. Mesmo observando que o p-valor para o coeficiente $\beta 3$ foi de 0,103 ao se realizar o teste de combinação linear da soma de $\beta 1, \beta 2$ e $\beta 3$, o p-valor foi 0,00 .

Tabela 4.2 - Efeito líquido da assimetria dos custos

\begin{tabular}{c|c|c|c|c}
\hline Variáveis & Coeficiente & Desvio Padrão & Estatística T & P-Valor \\
\hline Efeito do aumento receita & 0,7299 & 0,0110 & 5,80 & $0,000^{\star \star \star}$ \\
Efeito da diminuição receita & 0,5890 & 0,0182 & 32,27 & $0,000^{\star * \star}$ \\
\hline
\end{tabular}

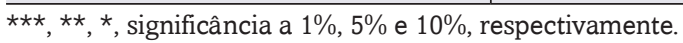

Fonte: Elaboração própria.

Na Tabela 5 é apresentada a estatística qui-quadrado do Teste de Hausman para o modelo I excluindo as empresas financeiras. Nota-se pelo p-valor que é rejeitada a hipótese nula de que a diferença de coeficientes estimados por meio de EF e EA é não sistemática, indicando que o estimador adequado é o de EF. Na Tabela 5.1, apresentam-se os resultados para o Modelo 1, excluindo-se as empresas financeiras, totalizando 6.222 observações. A magnitude do aumento nos custos e despesas de vendas e administrativas provenientes de um aumento na receita é superior à magnitude da diminuição nos custos e despesas de vendas e administrativas advindas de uma redução na receita. 
Tabela 5 - Teste de Hausman para modelo I excluindo as empresas financeiras

\begin{tabular}{c|c}
\hline & Coeficiente \\
\hline Chi-square test value & 6.2 \\
P-value & $0,045^{\star \star}$ \\
\hline
\end{tabular}

Esta tabela contém as regressões para todas as amostras.

***, **, *, significância a 1\%, 5\% e 10\%, respectivamente.

Fonte: Elaboração própria.

Observou-se que, quando se excluem as empresas financeiras, a magnitude do efeito se altera, porém, os custos continuam assimétricos. Portanto, isso não se rejeita a $\mathrm{H}_{1}$.

Tabela 5.1 - Análises do comp. Dos custos para modelo I excluindo as empresas financeiras

\begin{tabular}{|c|c|c|c|c|}
\hline Variáveis & Coeficiente & Desvio Padrão & Estatística $\mathrm{T}$ & P-Valor \\
\hline Receita & 0,7541 & 0,0105 & 71,28 & $0,000 * \star \star$ \\
\hline D1 & $-0,0706$ & 0,0190 & $-3,71$ & $0,000 * \star \star$ \\
\hline _Cons. & 0,0203 & 0,0029 & 6,83 & 0,000 \\
\hline \multicolumn{5}{|c|}{$\begin{array}{c}\text { Observances }=6.222 \\
\text { R-sq: } \text { Within }=0,6241 \\
\text { Between }=0,9483 \\
\text { Overall }=0,6351\end{array}$} \\
\hline
\end{tabular}

Esta tabela contém as regressões para todas as empresas, excluindo as empresas financeiras. CDVA são os custos despesas de venda e administrativa e é a variável dependente, receita são todas as receitas, D1 quando a receita diminui, variação do indicador tem valor de (1) quando a receita da empresa (i) no tempo ( $t$ ) diminui para período ( $t-1)$ e valor de (0) quando a receita aumenta.

***, **, *, significância a $1 \%, 5 \%$ e $10 \%$, respectivamente.

Fonte: Elaboração própria.

Desta forma, os resultados da presente pesquisa apontam que, quando a receita aumenta $1 \%$, os custos e despesas aumentam $0,75 \%$, porém, quando a receita diminui $1 \%$, esses mesmos custos e despesas reduzem $0,68 \%(0,7541-$ 0,0706), ou seja, os custos e despesas das empresas brasileiras possuem comportamento assimétrico. Esses resultados são similares aos encontrados por Richartz (2016), que identificou evidências de que as empresas brasileiras possuem comportamento assimétrico dos custos, similar aos encontrados na literatura internacional.

Tabela 5.2 - Efeito líquido da assimetria dos custos

\begin{tabular}{c|c|c|c|c}
\hline Variáveis & Coeficiente & Desvio Padrão & Estatística T & P-Valor \\
\hline Efeito do aumento receita & 0,7541 & 0,0105 & 71,28 & $0,000^{\star \star \star}$ \\
Efeito da diminuição receita & 0,6834 & 0,0133 & 51,19 & $0,000^{\star \star \star}$ \\
\hline
\end{tabular}

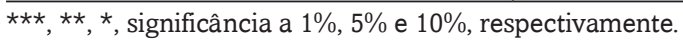

Fonte: Elaboração própria.

Na Tabela 6 é apresentada a estatística qui-quadrado do Teste de Hausman para o modelo II excluindo as empresas financeiras. Nota-se pelo p-valor que é rejeitada a hipótese nula de que a diferença de coeficientes estimados por meio de EF e EA é não sistemática, indicando que o estimador adequado é o de EF. Na Tabela 6, apresentam-se os resultados para o Modelo 2, também excluindo-se as empresas financeiras.

Tabela 6 - Teste de Hausman para modelo II adoção completa das IFRS, excluindo as empresas financeiras

\begin{tabular}{c|c}
\hline & Coeficiente \\
\hline Chi-square test value & 6,598 \\
P-value & $0,086^{\star}$ \\
\hline
\end{tabular}

Esta tabela contém as regressões para todas as amostras.

***, **, *, significância a 1\%, 5\% e 10\%, respectivamente.

Fonte: Elaboração própria. 
Observou-se que o aumento dos custos e despesas de vendas e administrativas para um aumento da receita é superior à diminuição dos custos e despesas de vendas e administrativas para uma redução da receita, com isso não se rejeita a $\mathrm{H}_{2}$.

Tabela 6.1 - Análises do comportamento dos custos para modelo II adoção completa das IFRS, excluindo as empresas financeiras

\begin{tabular}{|c|c|c|c|c|}
\hline Variáveis & Coeficiente & Desvio Padrão & Estatística T & P-Valor \\
\hline Receita & 0,7546 & 0,0105 & 71,31 & $0,000 \star \star \star *$ \\
\hline D1 & $-0,0947$ & 0,0233 & $-4,06$ & 0,000 *** \\
\hline D1*IFRS & 0,0461 & 0,0257 & 1,79 & $0,074^{\star}$ \\
\hline _Cons & 0,0203 & 0,0029 & 6,82 & 0,000 \\
\hline \multicolumn{5}{|c|}{$\begin{array}{c}\text { Observances }=6.222 \\
\text { R-sq.: Within }=0,6243 \\
\text { Between }=0,9468 \\
\text { Overall }=0,6354\end{array}$} \\
\hline
\end{tabular}

Esta tabela contém as regressões para todas as empresas, excluindo as empresas financeiras. CDVA são os custos despesas de venda e administrativa e é a variável dependente, receita são todas as receitas, D1 quando a receita diminui, variação do indicador tem valor de 1 quando a receita da empresa i no tempo t diminui para período $t-1$ e valor de 0 quando a receita aumenta, D1*IFRS após adoção das IFRS variação do indicador tem valor de 0 nos anos 2000 a 2009 e valor de 1 para os anos 2010 a 2016.

***, **, *, significância a $1 \%, 5 \%$ e $10 \%$, respectivamente.

Fonte: Elaboração própria.

Desta forma, os resultados apontam que, quando a receita aumenta $1 \%$, os custos e despesas aumentam $0,75 \%$, porém, quando a receita diminui $1 \%$, esses mesmos custos e despesas reduzem $0,71 \%(0,7546-0,0947+0,0461)$, valor esse maior do que observado na Tabela 6, sugerindo que os custos são menos pegajosos, sendo uma evidência contrária à encontrada por Bugeja et al. (2015), os quais, em análise das empresas australianas, identificaram que o comportamento dos custos é mais pegajoso após a adoção das IFRS.

Tabela 6.2 - Efeito líquido da assimetria dos custos

\begin{tabular}{c|c|c|c|c}
\hline Variáveis & Coeficiente & Desvio Padrão & EstatísticaT & P-Valor \\
\hline Efeito do aumento receita & 0,7546 & 0,0105 & 1,31 & $0,000^{\star \star \star}$ \\
Efeito da diminuição receita & 0,7059 & 0,0183 & 37,48 & $0,000^{\star \star \star}$ \\
\hline
\end{tabular}

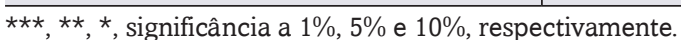

Fonte: Elaboração própria.

Na Tabela 7 é apresentada a estatística qui-quadrado do Teste de Hausman para o modelo I considerando somente as empresas financeiras. Nota-se pelo p-valor que é rejeitada a hipótese nula de que a diferença de coeficientes estimados por meio de EF e EA é não sistemática, indicando que o estimador adequado é o de EA.

Tabela 7 - Teste de Hausman para modelo I somente para as empresas financeiras

\begin{tabular}{c|c}
\hline & Coeficiente \\
\hline Chi-square test value & 0,746 \\
P-value & 0,689 \\
\hline
\end{tabular}

Esta tabela contém as regressões para todas as amostras.

$\star \star \star, ~ * \star$, *, significância a $1 \%, 5 \%$ e $10 \%$, respectivamente.

Fonte: Elaboração própria.

Na Tabela 7.1, apresentam-se os resultados para o Modelo 1, considerando somente as empresas financeiras, totalizando 376 observações. Desta forma, os resultados apontam que, quando a receita aumenta $1 \%$, os custos e despesas aumentam $0,62 \%$. Entretanto, quando a receita diminui $1 \%$, esses mesmos custos e despesas reduzem apenas $0,19 \%(0,6200-0,4290)$. Visto que há uma diferença apenas na magnitude do efeito, os custos e despesas das empresas financeiras brasileiras possuem comportamento assimétrico, mostrando-se mais pegajosos do que as demais empresas. Esse resultado pode ocorrer porque as empresas financeiras não possuem grandes custos fixos. 
Tabela 7.1 - Análises do comportamento dos custos para modelo I somente para as empresas financeira

\begin{tabular}{|c|c|c|c|c|}
\hline Variáveis & Coeficiente & Desvio Padrão & Estatística T & P-Valor \\
\hline Receita & 0,6200 & 0,0609 & 10,17 & 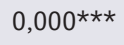 \\
\hline D1 & $-0,4290$ & 0,1137 & $-3,77$ & $0,000 * * *$ \\
\hline _Cons. & 0,0270 & 0,0305 & $-0,88$ & 0,377 \\
\hline \multicolumn{5}{|c|}{$\begin{array}{c}\text { Observances }=\quad 376 \\
\text { R-sq.: } \text { Within }=0,2848 \\
\text { Between }=0,2738 \\
\text { Overall }=0,2835\end{array}$} \\
\hline
\end{tabular}

Esta tabela contém as regressões somente para as empresas financeiras. CDVA são os custos despesas de venda e administrativa e é a variável dependente, receita são todas as receitas, D1 quando a receita diminui, variação do indicador tem valor de (1) quando a receita da empresa (i) no tempo ( $t$ ) diminui para período $(t-1)$ e valor de (0) quando a receita aumenta.

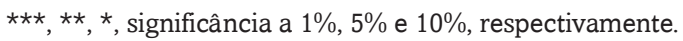

Fonte: Elaboração própria.

Observou-se que o aumento dos custos e despesas de vendas e administrativas para um aumento da receita é superior à diminuição dos custos e despesas de vendas e administrativas para uma redução da receita. Portanto, não se rejeita a $\mathrm{H}_{1 \mathrm{a}}$.

Tabela 7.2 - Efeito líquido da assimetria dos custos

\begin{tabular}{c|c|c|c|c}
\hline Variáveis & Coeficiente & Desvio Padrão & Estatística T & P-Valor \\
\hline Efeito do aumento receita & 0,6200 & 0,0609 & 10,17 & $0,000^{\star \star \star}$ \\
Efeito da diminuição receita & 0,1909 & 0,0764 & 2,50 & $0,012^{\star \star \star}$ \\
\hline
\end{tabular}

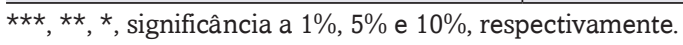

Fonte: Elaboração própria.

Na Tabela 8 é apresentada a estatística qui-quadrado do Teste de Hausman para o modelo II considerando somente as empresas financeiras. Nota-se pelo p-valor que é rejeitada a hipótese nula de que a diferença de coeficientes estimados por meio de EF e EA é não sistemática, indicando que o estimador adequado é o de EA.

Tabela 8 - Teste de Hausman para modelo II adoção completa das IFRS somente para as empresas financeiras

\begin{tabular}{c|c}
\hline & Coeficiente \\
\hline Chi-square test value & 1,961 \\
P-value & 0,581 \\
\hline
\end{tabular}

Esta tabela contém as regressões para todas as amostras.

$\star \star \star, ~ * \star$, *, significância a 1\%,5\% e $10 \%$, respectivamente.

Fonte: Elaboração própria.

Na Tabela 8.1, apresentam-se os resultados para o Modelo 2, considerando-se, também, somente as empresas financeiras. Desta forma, os resultados apontam que, quando a receita aumenta $1 \%$, os custos e despesas aumentam $0,62 \%$. Todavia, quando a receita diminui $1 \%$, esses mesmos custos e despesas reduzem apenas $0,20 \%(0,6205-0,4831$ $+0,0632$ ), valor esse maior do que foi observado na Tabela 8.1. 
Tabela 8.1 - Análises do comportamento dos custos para modelo II adoção completa das IFRS somente para as empresas financeiras

\begin{tabular}{|c|c|c|c|c|}
\hline Variáveis & Coeficiente & Desvio Padrão & Estatística T & P-Valor \\
\hline Receita & 0,6205 & 0,0610 & 10,17 & $0,000 * \star *$ \\
\hline D1 & $-0,4831$ & 0,1850 & $-2,61$ & $0,009 * \star \star$ \\
\hline D1*IFRS & 0,0632 & 0,1704 & 0,37 & 0,711 \\
\hline _Cons & $-0,0273$ & 0,0306 & 0,89 & 0,371 \\
\hline \multicolumn{5}{|c|}{$\begin{array}{c}\text { Observances }=376 \\
\text { R-sq.: Within }=0,2844 \\
\text { Between }=0,2972 \\
\text { Overall }=0,2838\end{array}$} \\
\hline
\end{tabular}

Esta tabela contém as regressões somente para as empresas financeiras. CDVA são os custos despesas de venda e administrativa e também a variável dependente, receita são todas as receitas, D1 quando a receita diminui, variação do indicador tem valor de 1 quando a receita da empresa i no tempo t diminui para período t -1 e valor de 0 quando a receita aumenta, D1*IFRS após adoção das IFRS variação do indicador tem valor de 0 nos anos 2000 a 2009 e valor de 1 para os anos 2010 a 2016.

***, **, *, significância a 1\%, 5\% e 10\%, respectivamente.

Fonte: Elaboração própria.

Observou-se que o aumento dos custos e despesas de vendas e administrativas para um aumento da receita é superior à diminuição dos custos e despesas de vendas e administrativas para uma redução da receita, com isso não se rejeita a $\mathrm{H}_{2}$. Mesmo observando que o p-valor para o coeficiente $\beta 3$ foi de 0,711 ao se realizar o teste de combinação linear da soma de $\beta 1$, $\beta 2$ e $\beta 3$, o p-valor foi 0,013 .

Tabela 8.2 - Efeito líquido da assimetria dos custos

\begin{tabular}{c|c|c|c|c}
\hline Variáveis & Coeficiente & Desvio Padrão & Estatística T & P-Valor \\
\hline Efeito do aumento receita & 0,6205 & 0,0610 & 10,17 & $0,000^{\star \star \star}$ \\
Efeito da diminuição receita & 0,2005 & 0,0807 & 2,48 & $0,013^{\star \star}$ \\
\hline
\end{tabular}

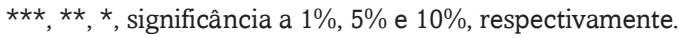

Fonte: Elaboração própria.

\section{CONCLUSÃO}

A pesquisa teve como objetivo principal analisar se a adoção completa das IFRS impactou no comportamento assimétrico dos custos e, para isso, foram analisadas todas as empresas brasileiras listadas na B3, o que resultou em 6.598 observações.

Os resultados encontrados, quando não considerada a adoção das IFRS, indicam um aumento da receita de $1 \%$, os custos e despesas aumentam $0,73 \%$ e, quando a receita diminui em 1\%, os custos e despesas reduzem 0,61\%. Tais resultados já eram esperados, conforme visto na revisão da literatura nacional e internacional, quando comparado com estudo de Richartz (2016), que aponta que, quando a RLV aumenta 1\%, os custos totais aumentam 0,74\%. Contudo, quando a RLV reduz $1 \%$, esses mesmos custos reduzem apenas $0,68 \%$. Os resultados de Anderson et al. (2003) mostram que os custos crescem em média 0,55\% para um aumento de $1 \%$ na receita líquida de vendas, mas diminuem somente 0,35\% para uma redução de 1\% na receita líquida de vendas. Além disso, Banker \& Byzalov (2014) afirmam que o comportamento assimétrico dos custos é um fenômeno global.

Porém, quando se considera a adoção das IFRS, os resultados apontam que, quando a receita aumenta $1 \%$, os custos e despesas aumentam $0,73 \%$, mas, quando a receita diminui $1 \%$, os custos e despesas reduzem $0,59 \%$. Esse valor é menor do que o que foi observado anteriormente, apresentando um resultado mais pegajoso, o que também indica a similaridade com a literatura internacional já existente.

Foram analisados, também, excluindo-se as empresas financeiras, tendo sido contadas 6.222 observações sem considerar adoção das IFRS. Os resultados apontam que, quando a receita aumenta 1\%, os custos e despesas aumentam $0,75 \%$. Entretanto, quando a receita diminui $1 \%$, esses mesmos custos e despesas reduzem $0,68 \%$. Por outro lado, considerando a adoção das IFRS, os resultados apontam que, quando a receita aumenta 1\%, os custos e despesas aumentam $0,75 \%$. Contudo, quando a receita diminui $1 \%$, esses mesmos custos e despesas reduzem $0,71 \%$. Tal valor é maior do que foi encontrado anteriormente. Portanto, os dados mostram que os custos são menos pegajosos, trazendo resultado diferente do encontrado na literatura internacional, bem como no estudo de Bugeja et al. (2015), que pesquisaram as empresas australianas e observaram um comportamento em que os custos aumentaram $0,88 \%$ com um aumento de $1 \%$ em receitas de vendas, mas diminuíram apenas 0,80\% para uma queda de $1 \%$ nas receitas de vendas. 
Além disso, foram analisadas somente as empresas financeiras, contando com 376 observações, e os resultados apontam que, quando a receita aumenta $1 \%$, os custos e despesas aumentam $0,62 \%$. Todavia, quando a receita diminui $1 \%$, esses mesmos custos e despesas reduzem apenas $0,19 \%$. Ao considerar a adoção das IFRS, os resultados apontam que, quando a receita aumenta $1 \%$, os custos e despesas aumentam $0,62 \%$. Contudo, quando a receita diminui $1 \%$, esses mesmos custos e despesas reduzem apenas 0,20\%. Tal valor é maior do que o observado anteriormente. Portanto, observa-se que os custos são menos pegajosos. Além disso, observou-se que os custos das empresas do setor financeiros não variam na mesma intensidade das receitas, pois apresentam um custo bem mais stick do que o normal. Esse resultado pode ocorrer porque as empresas financeiras possuem uma legislação própria, além de mais custos e despesas fixas, contribuindo assim com um custo mais pegajoso.

A presente pesquisa conclui que as empresas brasileiras possuem comportamentos assimétricos dos custos, resultado esse similar aos já existentes na literatura nacional e internacional. Agora, quando considera a adoção completa das IFRS para todas as empresas, o resultado apresenta um comportamento dos custos mais pegajoso. Quando se analisa, excluindo as empresas financeiras ou considerando somente as empresas financeiras, o resultado apresenta um comportamento dos custos menos pegajoso.

Tais resultados encontrados após a adoção das IFRS trazem indícios de que se aumentou o comportamento assimétrico dos custos, sendo essa informação crucial para o processo de tomada de decisão dos investimentos. Por consequência, é também de suma importância para conhecimentos dos investidores e analistas de mercado, que devem considerar em suas análises a estrutura dos custos fixos, uma vez que são considerados custos pegajosos.

Por fim, a pesquisa atingiu seu objetivo que é contribuir com a teoria sobre comportamento dos custos após a adoção das IFRS. Como limitação da pesquisa, pode-se destacar a falta de literatura sobre o assunto pesquisado, mas com oportunidades de ampliação desse campo do conhecimento. Ademais, sugerem-se futuras pesquisas, para o aprofundamento sobre o tema, como separar por setores, assim identificando qual setor pode ter obtido maior influência da IFRS sobre assimetria dos custos. Os estudos futuros podem, ainda, explorar outros fatores que impactam na assimetria dos custos, como por exemplo, o gerenciamento de resultados, a agressividade tributária e o quão intensiva uma empresa é em investimentos em imobilizados. Tratando especificamente do contexto da adoção do IFRS no Brasil, os trabalhos podem ainda abordar o efeito de cada CPC publicado e o seu real impacto no reconhecimento das receitas e das despesas. Outros dois pontos ainda não abordados na literatura nacional são o efeito das crises e o controle de empresas por parte do governo. Por fim, seria interessante a realização de estudos cross-country bem como a análise do impacto da governança corporativa, por meio dos níveis de governança da B3 e de empresas listadas.

\section{REFERÊNCIAS}

Anderson, M. C., Banker, R. D., \& Janakiraman, S. N. (2003). Are selling, general and administrative costs "sticky"? Journal of accounting research, 41(1), 47-63.

Anderson. S. W. (2006). Managing costs and cost structure throughout the value chain: research on strategic cost management forthcoming. In: Chapman, C.; Hopwood, A; \& Shields, M. Handbook of management accounting research. (Vol. 2). Oxford: Elsevier.

Balakrishnan, R., Labro, E., \& Soderstrom, S. (2014). Cost structure and sticky costs. Journal of management accounting research, 26(2), 91-116.

Banker, R. D.; \& Byzalov, D. (2014). Asymmetric cost behavior. Journal of management accounting research american, $26(2), 43-79$.

Banker, R. D.; Byzalov, D.; \& Chen, L. (2013). Employment protection legislation, adjustment costs and cross-country differences in cost behavior. Journal of accounting and economics, 55(1), 111-127.

Banker, R. D., Byzalov, D.; Ciffci, M., \& Mashruwala, R. (2014). The moderating effect of prior sales changes on asymmetric cost behavior. Journal of management accounting research, 26(2), 221-242.

Bugeja, M., Lu, M., \& Shan, Y. (2015). Cost Stickiness in Australia: characteristics and determinants. Australian accounting review, 25(3), 248-261.

Calleja, K., Steliaros, M., \& Thomas. D. C. (2006). A note on cost stickiness: some international comparisons. Management accounting research, 17, 127-140.

Chen, C. X., Lu, H., \& Sougiannis, T. (2008). Managerial empire building, corporate governance, and the asymmetrical behavior of selling, general, and administrative costs. Anais do AAA Financia

l Accounting and Reporting Section (FARS) Paper and (CAAA) 2008 Annual Conference Paper, University of Toronto and University of Illinois at Urbana-Champaign, Illinois, Estados Unidos.

Comitê de pronunciamentos contábeis pronunciamento técnico - CPC 27 Ativo Imobilizado, (2009).Recuperado de : http://www. cpc.org.br/CPC/Documentos-Emitidos/Pronunciamentos

Comitê de pronunciamentos contábeis pronunciamento técnico - CPC 16 (R1) Estoques, (2009). Recuperado de : http://www. cvm.gov.br/export/sites/cvm/menu/regulados/normascontabeis/cpc/CPC_16_R1_rev_12.pdf

Dierynck, B., Landsman, W.R., \& Renders, A. (2012) Do Managerial Incentives drive Cost Behavior? Evidence about the Role of the Zero Earnings Benchmark for Labor Cost Behavior in Belgian Private Firms. The Accounting Review, 87(4), 1219-1246. doi: https://doi.org/10.2308/accr-50153 
Domingos, S. R. M., Lima, S. M., \& Ponte, V. M. R. (2013). Income Smoothing: um estudo após a adoção do IFRS no Brasil. Revista contemporânea de economia e gestão. 11(2), 181-199.

Elias, T. M., Borgert, A., \& Richartz, F. (2014). A influência dos gastos com mão de obra na assimetria dos custos das empresas brasileiras listadas na B3. Anais do XXI Congresso Brasileiro de Custos. Natal, RN, Brasil, 21.

Gatsios, R. C., Silva, J. M., Ambrozini, M. A., Assaf, A. A., Neto, \& Lima, F. G. (2016). Impacto de adoptar la NIIF sobre el costo de capital de las empresas que figuran en Brasil. RAM - Rev. Adm. Mackenzie, 17(4), 85-108.

Grejo, L. M., Santos, A., \& Abbas, K. (2015). Análise do Comportamento dos Custos em Empresas de Tecnologia da Informação Listadas na B3. Anais do $9^{\circ}$ Congreso Iberoamericano de Contabilidad de Gestión, Florianópolis, SC, Brasil, 1.

Kama, I.; \& Weiss, D. (2013). Do earnings targets and managerial incentives affect sticky costs? Journal of Accounting Research, 51(1), 201-224.

Lourenço, I. M. C., \& Branco. M.E.M.A.D.C. (2015). Principais consequências da adoção das IFRS: análise da literatura existente e sugestões para investigação futura. Revista Contabilidade \& Finanças, 26(68), 126-139.

Medeiros, O. R., Costa, P. de S., \& Silva, C. A. T. (2005). Testes empíricos sobre o comportamento assimétrico dos custos nas empresas brasileiras. Revista Contabilidade \& Finanças, 16(38), 47-56.

Porporato, M., \& Werbin, E. M. (2010). Active Cost Management in Banks: Evidence of Sticky Costs in Argentina, Brazil and Canada. Anais do AAA 2011 Management Accounting Section (MAS) Meeting Paper. Recuperado em 26 fevereiro, 2019, de https://ssrn.com/ abstract=1659228 ou http://dx.doi.org/10.2139/ssrn.1659228 .

Richartz, F., Borgert, A., \& Lunkes, R. J. (2014). Comportamento assimétrico dos custos nas empresas brasileiras listadas na B3. Scientific And Applied, 7(3), 339-361.

Richartz, F. (2016). Fatores explicativos para o comportamento assimétrico dos custos das empresas brasileiras. (Tese de Doutorado). Universidade Federal de Santa Catarina, Florianópolis, SC, Brasil.

Santos, M. A. C., \& Cavalcante, P. R. N. (2014). O efeito da adoção dos IFRS sobre a relevância informacional do lucro contábil no Brasil. Revista Contabilidade \& Finanças., 25(66), 228-241.

Silva; M. P.; Bonfim; J. K.; \& Niyama; C. A. T. (2017). Adoção ao padrão IFRs e earnings quality: a persistência do lucro das empresas listadas na B3. Revista de Contabilidade e Organizações, 11(29), 46-55. Recuperado em 26 fevereiro, 2019, doi: http://dx.doi. org/10.11606/rco.v11i29.125846.

Silva, T. D., \& Miranda, G. J. (2016). Os indicadores relativos à gestão do capital de giro antes e depois da adoção dos padrões internacionais de contabilidade no Brasil. Revista de educação e pesquisa em contabilidade, 10(3), 258-271.

Silva, A. da, Zonatto, V. C. da S., Magro, C. B. D., \& Klann, R. (2019). Sticky Costs Behavior and Earnings Management. Brazilian Business Review, 16(2), 191-206. https://doi.org/10.15728/bbr.2019.16.2.6

Sousa, E. F., Sousa, A. F., \& Demonier, G. B. (2016). Adoção das IFRS no Brasil: efeitos no conservadorismo contábil. Repec - Revista De Educação E Pesquisa Em Contabilidade, 10(2), 136-147.

Telles. S. V., \& Salotti. B. M. (2015). Divulgação da informação contábil sobre depreciação: o antes e o depois da adoção das IFRS. Revista Universo Contábil, 11(2), 153-173.

Weiss, D. (2010). Cost behavior and analysts' earnings forecasts. The Accounting Review, 85(4), 1441-71. 